\title{
Association between beverages consumption and total diet quality index with sedentary behaviours in Spanish children. Calina study
}

\author{
María L. Miguel-Berges ${ }^{1}$, Alba Santaliestra-Pasias ${ }^{1,2}$, Iris Iglesia-Altaba ${ }^{1,3}$, \\ Paloma Flores-Barrantes ${ }^{1}$, Pilar Samper ${ }^{1}$, Luis A. Moreno ${ }^{1,2}$ and Gerardo Rodriguez ${ }^{1,3}$ \\ ${ }^{1}$ Growth, Exercise, Nutrition and Development (GENUD) Research Group, University of Zaragoza, Instituto \\ Agroalimentario de Aragón (IA2), Zaragoza, Spain, \\ ${ }^{2}$ Centro de Investigación Biomédica en Red de Fisiopatología de la Obesidad y Nutrición (CIBERObn), Zaragoza, \\ Spain and \\ ${ }^{3}$ Red de Salud Materno-infantil y del Desarrollo (SAMID), Zaragoza, Spain
}

\begin{abstract}
Introduction: Evidence suggests that the time spent in different types of sedentary behaviours (e.g. TV watching, use of video games) may be more important in predicting childhood obesity as compared with overall sedentary time (ST). Sedentary time has been associated with unhealthy food and beverage consumption at young ages. The aims of this study were: to examine the association between different beverages consumption and sedentary behaviours and to evaluate the association between the total dietary quality index (DQI) and the adherence to the ST recommendations, in a sample of Spanish children.
\end{abstract}

Methodology: The study included a cohort of 381 children (40.7 \% boys) aged 7 years, from the Growth and Feeding during Infancy and Early Childhood in Aragon (CALINA) longitudinal study, living in Zaragoza. Data on dietary habits and screen time (watching TV/DVD/videos and playing PC/video games) were parental-reported. ST was categorized based on the recommendations $(\leq 2 \mathrm{hour} /$ day and $>2$ hour/day). The DQI was computed from a validated semi quantitative food-frequency questionnaire. Generalized linear mixed models, adjusted for maternal education and body mass index, were analysed.

Results: In boys, exceeding the total ST's recommendations ( $>2 \mathrm{~h} /$ day) was positively associated with high consumption of soft drinks $(\beta=0.13$; CI: $0.02 ; 0.26)$ while in girls, exceeding the recommendations was positively associated with light drinks (soft drinks with artificial sweeteners) consumption $(\beta=0.06$; CI: $0.02 ; 0.13)$. Only individual PC/videogames time was associated with beverage consumption. In both gender, excessive PC/video games time ( $>2 \mathrm{~h} /$ day) was associated with high soft drinks consumption $(\beta=0.51$; CI: $0.25 ; 0.77, \beta=1.07$; CI: $0.79 ; 1.35$, respectively). Only in girls, there was a positive association between PC/ video games time and light drinks (soft drinks with artificial sweeteners) consumption $(\beta=0.21$; CI: $0.02 ; 0.41)$. Regarding the DQI, only boys showed a negative associations between total ST and the DQI $(\beta=-5.91$; CI $-2.56 ;-2.56)$, this means, that those boys that exceeded the total ST's recommendations ( $>2 \mathrm{~h} /$ day) had a poor total quality diet.

Discussion: In children, ST was associated with beverages (soft drinks and light drinks) consumption and a poor total diet quality. Soft drinks with artificial sweeteners (light drinks) were chosen mostly by girl's, whereas, boys preferred regular sugar sweetened soft drinks consumption.

\section{Conflict of Interest}

There is no conflict of interest 